\title{
Erratum to: Models of cardiac electromechanics based on individual hearts imaging data
}

\section{Image-based electromechanical models of the heart}

\author{
Viatcheslav Gurev • Ted Lee • Jason Constantino • \\ Hermenegild Arevalo • Natalia A. Trayanova
}

Published online: 9 January 2011

(C) Springer-Verlag 2011

\section{Erratum to: Biomech Model Mechanobiol}

\section{DOI 10.1007/s10237-010-0235-5}

Equations (2) and (3) should be replaced, respectively, with $\left\{\begin{array}{l}A_{1} \oplus A_{2}=\exp \left(\ln \left(A_{1}\right)+\ln \left(A_{2}\right)\right) \\ k \otimes A=A^{k}=\left(U D U^{T}\right)^{k}=U D^{k} U^{T}=U\left[\begin{array}{ccc}\lambda_{1}^{k} & 0 & 0 \\ 0 & \lambda_{2}^{k} & 0 \\ 0 & 0 & \lambda_{3}^{k}\end{array}\right] U^{T},\end{array}\right.$

and

$$
A=\exp \left(\sum_{i} \psi_{i} \times \ln \left(A_{i}\right)\right) .
$$

Simulation results in the paper are not affected by this error since the correct expressions were used in generating the results.

In Sect. 2.6, first paragraph, the sentence "In this semiimplicit scheme, $x_{k+1}$ (the value of $\mathrm{x}$ at the next integration step) was used for extrapolation of $\frac{\mathrm{d} x}{\mathrm{~d} t}$ and for integration of the first equation in (7) ..." should read as follows: "In this semi-implicit scheme, $x_{k+1}$ (the value of $\mathrm{x}$ at the next integration step) was used for extrapolation of $\frac{\mathrm{d} x}{\mathrm{~d} t}$ and for integration of the first equation in (8) ...".

The online version of the original article can be found under doi:10.1007/s10237-010-0235-5.

V. Gurev $(\bowtie) \cdot$ T. Lee $\cdot$ J. Constantino $\cdot$ H. Arevalo $\cdot$ N. A. Trayanova Institute for Computational Medicine, Department of Biomedical Engineering, Johns Hopkins University, 3400 N. Charles St., CSEB Room 218, Baltimore, MD 21218, USA

e-mail:vgurev@jhu.edu; vgurev@gmail.com 